\title{
Effect of sampling strategy on uncertainty and precision of flatness inspection studied by dynamic minimum deviation zone evaluation
}

A. Barari` and S. Mordo

Department of Automotive, Mechanical, and Manufacturing Engineering, Faculty of Engineering and Applied Science, University of Ontario Institute of Technology, Oshawa, Ontario, Canada L1H 7K4

Received: 23 November 2012 / Accepted: 28 November 2012

\begin{abstract}
Evaluation of geometric deviations for the purpose of determining compliance with specified form tolerances requires the acquisition of numerous measured data points and extensive computation to accurately characterize the inspected part's geometry. If there are not enough data points measured or when the measured points are not distributed properly on the measured surface a high level of uncertainty in characterizing the inspected part's geometry can be expected. However, increasing the number of data point also significantly increases the computational time and also increases computational uncertainty by adding to instability of the optimization process required to find the minimum deviation zone. Selections of number and location of the measured date points need to be performed by understanding the significance of these two sources of uncertainties. This paper discussed the effect of sampling procedure on uncertainty and precision of flatness inspection.
\end{abstract}

Keywords: Coordinate metrology; dynamic minimum zone evaluation; minimum deviation zone; measurement uncertainty; convex hull

\section{Introduction}

The examination of compliance with specified geometric and form tolerances requires acquisition of numerous measured data points and extensive computation. This process is required to accurately characterize the inspected part's geometry. Traditionally, the required computation and the software analyses are performed in three major sequential tasks. The first task is to develop a data sampling plan. The sampling plan needs to be developed based on the workpiece's characteristics and also type of the employed measurement equipment. The number and locations of surface measurement points are amongst the most important parameters that influence the accuracy and validation of the whole assessment process. However, optimum determination of these parameters is often a difficult and challenging process and no standard procedure or guideline has been developed for it yet.

Next, conformance of the actual geometry to the desired tolerances is evaluated by fitting a substitute geometry to the data points captured by a Coordinate Measuring Machine (CMM).

The third task is to calculate the geometrical deviations associated with the measured part. This task needs to be conducted based on the deviations of the data points from the best substitute geometry and to estimate the uncertainty of the assessed inspection results. Numerous research projects in the area of evaluating the geometric

* Correspondence: ahmad.barari@uoit.ca deviations based on the discrete measured points have been undertaken. However, the partitioning of these three key tasks is the prevailing theme in most of the reported approaches. Alternatively, these three tasks can be performed concurrently with continuous feedbacks or as the elements of a closed-loop between the three tasks. This integration and communication between the different modules enhances the level of certainty, which results in more reliable decisions made by each individual task. In a closed-loop system, an estimation of the form and nature of the geometric deviations can be used to acquire the most useful data-set from the part. Knowing the characteristics of this data-set improves the estimation of the optimum substitute geometry and reduces the corresponding computation cost. Also, knowledge of the fitting criteria and its procedure results in accurate estimation of the geometric deviation and reduces uncertainty of the inspection decisions.

This paper discusses how severely the decisions made for the first task (sampling plan) will affect the uncertainty of evaluation process. In order to study this effect the problem is solved for the case of flatness analyses. Flatness evaluation is selected for this study because there is much less uncertainty in its required computational process related to the second task, i.e., fitting a substitute geometry to the data points. A Convex-Hull approach is adapted to evaluate exact Minimum Deviation Zone (MDZ) of the captured data points. Without being concerned of the possible uncertainties due to the fitting 
process, an examination platform is developed to purely study the significance of uncertainty due to the employed sampling plan. Also using convex-hull properties in evaluation of MDZ enabled us to utilize adopting a Dynamic Minimum Deviation Zone (DMDZ) evaluation approach. DMDZ evaluation allows a loop between the first two computational tasks, i.e., sampling and the fitting processes.

By conducting statistical analyses on the experimental data interesting results are achieved that demonstrates the level of reliability of traditional coordinate metrology processes in inspection of the ordinary manufactured parts and surfaces.

\section{Theoretical background}

Coordinate Measuring Machines are used to acquire discrete points from the surface of manufactured parts. After surface point measurement, the conformance of the actual surface to the desired tolerance is evaluated by fitting a substitute geometry to the measured points. Fitting is achieved by minimizing an error function between the acquired points and the substitute geometry. Simplification and approximation methods are utilized by most fitting algorithms to simplify the problem and speed up computation. In addition, many CMM manufacturers implement their own proprietary programs without explicitly stating the underlying assumptions and limitations of their algorithms.

Fitting substitute geometry to the data points by minimizing an error objective function is defined by the $L p$ norm equation [1]:

$$
L_{p}=\left[\frac{1}{n} \sum_{i}^{n}\left|\boldsymbol{r}_{i}\right|^{p}\right]^{1 / p}
$$

where $\boldsymbol{r}_{\mathbf{i}}$ is the vector of the residual error between the $i$ th measured point and the substitute geometry fitted to the $n$ data points, $i$ is the index of the measured point, and $p$ is an exponent. Least square $(p=2)$ and MDZ functions $(p=\infty)$ are typically utilized in the fitting process. The criterion in the least square function requires that the sum of square errors to be minimized. The least square best fit is the most likelihood estimation used to fit the substitute geometry onto a set of discrete data. Since all the measurement points contribute to the best-fit result, the substitute geometry is more stable and less sensitive to the local deviations, asperities and local surface effects. However, least square best fit is a statistical estimation rather than an exact solution and some concerns always exist regarding interpretation of its fitting results. For the MDZ function, equation (1) becomes [2]:

$$
L_{\infty}=\max _{i}\left(\left|\boldsymbol{r}_{i}\right|\right) \text {. }
$$

The MDZ function has received a great deal of attention in recent years, because the studies show that it yields a smaller zone value than that evaluated by using least squares fit [3]; and it best conforms to the standard definition of the tolerance zone in ASME Y14.5. In addition, it numerically simulates the physical fitting process in traditional metrology. However, such an extreme fit has limitations in accuracy [4] and stability [2]. The reason is that minimization of the deviation zone is a highly non-linear problem. Since the analytical derivatives of the objective function are not usually available, a direct search method is required to solve the problem and its success is very dependent on the search's initial conditions [4].

Research on coordinate sampling is mostly focused on the sample size and not the sample locations. Different sampling techniques, including uniform sampling, random sampling and stratified sampling [5], have been studied. Extensive numerical experiments on various geometric primitives, such as line, plane, circle, sphere and cylinder conclude that inspection results significantly vary by sampling size [4-6]. Weckenmann et al. observed that the evaluation accuracy is increased with increasing the sample size for circular and linear features and also concluded that different evaluation criteria call for different sampling strategies [7]. The distribution of the form errors on the actual geometry for flat surfaces was found to be critical for estimating the sample size as well as the uncertainty associated with the evaluation [4]. An iterative sampling method was proposed by Edgeworth and Wilhelm [8]. It is developed for CMMs capable of measuring the surface normal vectors in addition to the coordinate points. Using the available coordinate points and the normal vectors an algorithm interpolates the surface error between the measurement locations by a cubic polynomial and decides when and where additional sampling is required.

The concept of an integrated inspection system is implemented for inspection of sculptured surfaces by Barari et al. [2]. An integrated inspection system is developed based on the iterative search procedure and online least square estimation of geometric deviations. A pattern recognition technique called Parzen Windows is utilized for this purpose. Studying the second order discontinuities of the density function identifies the critical portions of the surface that require further measurement. The current paper presents an extension of this approach with focus on the on-line estimation of MDZ and studies the effect of the proposed integration on the uncertainty of the MDZ evaluation. In order to study the MDZ uncertainty several sets of experiments, including numerical experiments that use actual measurement data as well as experiments that use virtual measurement data are conducted. By analytical and statistical analyses of the results, it is shown that significant improvements in the accuracy of the entire tolerance evaluation process and certainty of the inspection are achieved. Although the integrated model is beneficial for different applications in coordinate metrology, the results emphasize that in the case of MDZ evaluation, using an adaptive sampling plan is beneficial.

\section{Experimental platform and data analyses}

Let $\boldsymbol{S}$ be a set of points in $E^{3}$. The convex hull $\boldsymbol{H}(\boldsymbol{S})$ is the smallest convex set containing entire $\boldsymbol{S}$. A supporting 
plane $\boldsymbol{\Psi}$ of $\boldsymbol{H}(\boldsymbol{S})$ is a plane passing through at least one vertex of $\boldsymbol{H}(\boldsymbol{S})$ such that the all the other points of convex set $\boldsymbol{H}(\boldsymbol{S})$ lie on one side of the half-space defined by $\boldsymbol{\Psi}$. Let $\boldsymbol{Z}(\boldsymbol{S})$ be a zone defined as follows:

$$
\boldsymbol{Z}(\boldsymbol{S})=\left\{x \in E^{3} \wedge \boldsymbol{\Psi}_{1} \leqslant x \leqslant \boldsymbol{\Psi}_{2}\right\}
$$

where $\boldsymbol{\Psi}_{1}$ and $\boldsymbol{\Psi}_{2}$ are two supporting parallel planes. Diameter $d$ of $\boldsymbol{Z}(\boldsymbol{S})$ is the distance between the two supporting parallel planes. There are many such zones can be defined and the Minimum Deviation Zone is the zone with the smallest diameter [9].

This definition is employed in evaluation of flatness MDZ of a set of sample points. A three-dimensional convex hull $\boldsymbol{H}(\boldsymbol{S})$ is generated using Delaunay Triangulations of the set of points [10]. The minimum zone of the set of sample points is then determined by finding the minimum zone of their convex hull. This is conducted by finding the minimum distance between a pair of parallel planes containing the convex hull [9]. By implementing this approach in our flatness evaluation, the minimum deviation zone is calculate accurately for all sets of sampling without including the typical computational uncertainties that exist in typical optimization-based fitting processes [2].

Flatness MDZ evaluation using convex hull properties also allows adopting a Dynamic Minimum Deviation Zone Evaluation procedure. This allows to conduct sampling iteratively and parallel to the MDZ evaluation process. The DMDZ evaluation is developed based on dynamic modification of the convex hull $\boldsymbol{H}(\boldsymbol{S})$ considering the newly measured sample point.

A typical manufactured flat surface with overall dimensions of $330 \mathrm{~mm}$ by $150 \mathrm{~mm}$ was measured by a CMM equipped with a laser scanning probe. Total of 148772 data points are measured from the entire surface using stratified sampling. Considering the significant number of data points, the detailed information of the measure surface could be generated. However, the computational time for MDZ evaluation of this amount of data is highly expensive and it is not practical for industrial application. In typical applications, usually a much smaller set of data points are randomly selected from the measured surface. However, reducing the amount of data can be interpreted as increasing uncertainty of the inspection process. In order to study the significance of this source of uncertainty nine different sets of 200 data points from different locations of the measured surface are randomly sampled. The sampling processes are virtually conducted by sampling from the original data points measured from the part's surface. Very high density of the original data points allows us to assume almost data of any small neighbourhood of surface points are available for virtual sampling.

For each set of sample points the MDZ is evaluated using an optimization-based fitting algorithm and also using the described convex hull approach. The deviation of two results for each set of sample points demonstrates the uncertainty of the utilized optimization-based fitting process. However, the deviations of evaluated MDZs for the nine different sample sets demonstrate the effect of sampling process on the inspection uncertainty. At the end,
Table 1. MDZ and normal vector of the optimum substitute plane for 148722 data points.

\begin{tabular}{cccc}
\hline MDZ & $\begin{array}{c}\text { Normal vector } \\
\text {-component }\end{array}$ & $\begin{array}{c}\text { Normal vector } \\
y \text {-component }\end{array}$ & $\begin{array}{c}\text { Normal vector } \\
z \text {-component }\end{array}$ \\
\hline $\pm 0.1164 \mathrm{~mm}$ & -0.0082 & 0.0044 & -0.9999 \\
\hline
\end{tabular}

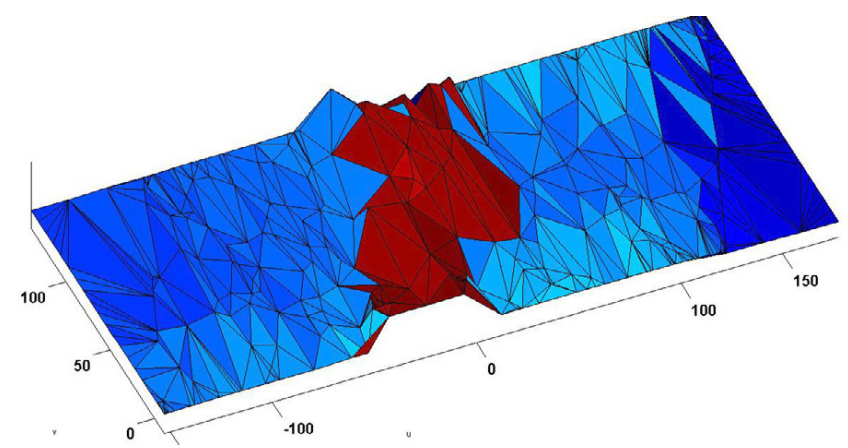

Fig. 1. Geometric form details of the inspected surface with $10 \times$ magnification.

the DMDZ procedure is used to develop the convex hull of the entire measured data points. The last result is used as the base for measuring effectiveness of the small-sized sampling approaches.

\section{Results and discussions}

The DMDZ evaluation procedure is conducted to gradually include all 148772 data points in developing the convex hull. The convex hull of the entire data points is calculated by a convex set of 184 points. By calculating the MDZ of the convex hull of the entire data points the details of surface are understood. Table 1 presents the inspection results including the MDZ and the normal vector of the optimum substitute geometry. Figure 1 demonstrates the details of the evaluated surface using triangulations. Form deviations are magnified with a factor of 100 in this picture for a better illustration.

Figure 2 presents the pattern of form deviations with a color-map technique. As can be seen, the evaluate minimum deviation zone is $0.2328 \mathrm{~mm}$.

The virtual sampling process is conducted using the random selection of sets of 200 data sets from the original date points. For each sampling the MDZ is evaluated using the described convex hull approach and using a typical optimization-based fitting algorithm.

Figure 3 shows an example of 200 virtually measured points in one of the experiments (sample set \#6) and the corresponding best fitted plane found by the described convex hull approach. Points with negative deviations are shown with two concentric blue circles while points with positive deviations are shown with Red color single circles. 


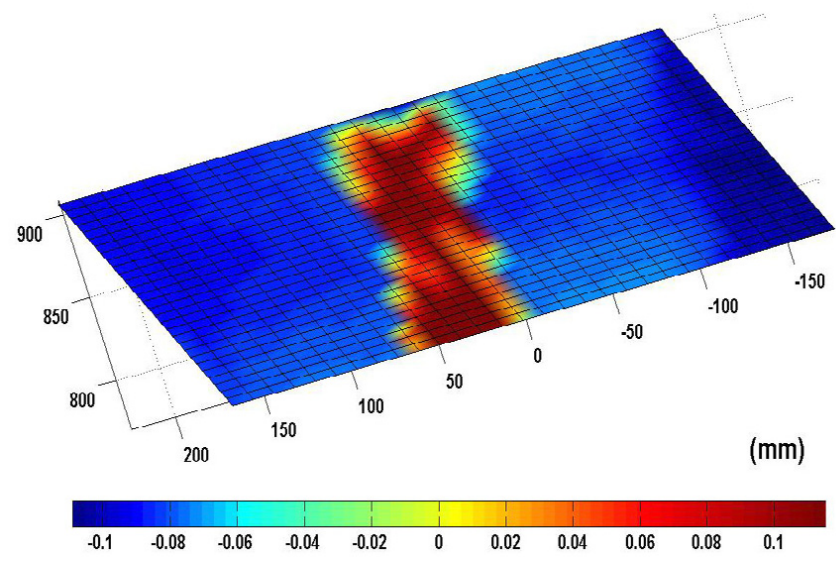

Fig. 2. Pattern of form deviations developed by MDZ evaluation of 148772 data points.

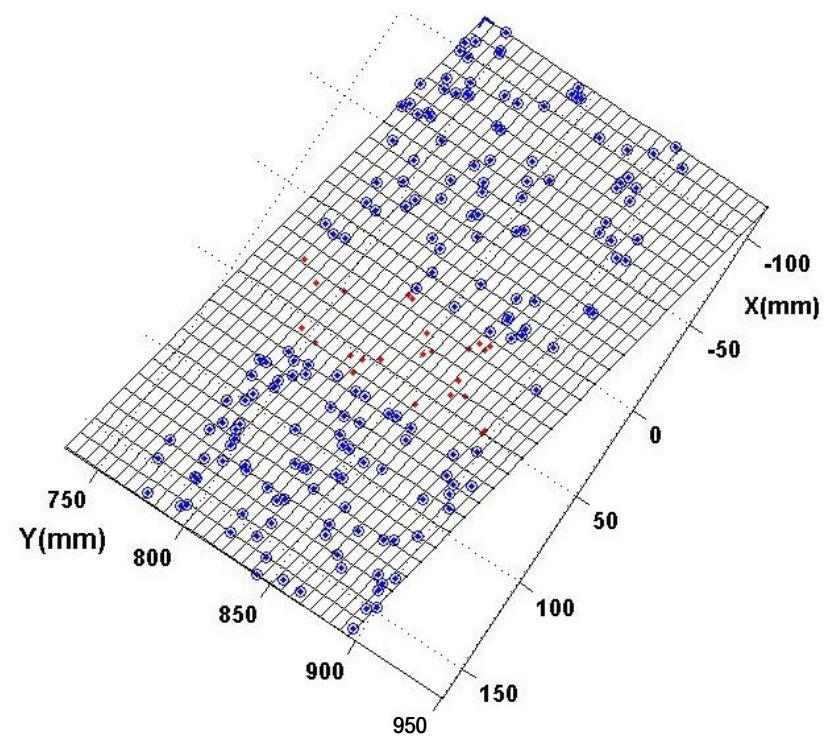

Fig. 3. Randomly selected 200 measured points in one of the experiment (sample set \#6) and the optimum substitute plane evaluated by the convex hull approach.

Figure 4 shows the evaluated MDZ for three experiments using different sets of 200 randomly sampled points (sets 1, 7 and 9, respectively). The observed differences in the recognized patterns demonstrated in the figures by color maps.

The results of MDZ evaluations for nine different random sampling processes from the original 148772 data points are presented in Table 2. The MDZs for each case are evaluated using the described convex hull method and the optimum substitute plane is calculated. The normal vectors of the optimum substitute planes are presented also in this table. The inspection errors are calculated as the percentage of difference with the exact solution evaluated by DMDZ evaluation of the entire original data points.

The first important observation is the uncertainty of the evaluated MDZ based on the sampling. As can be

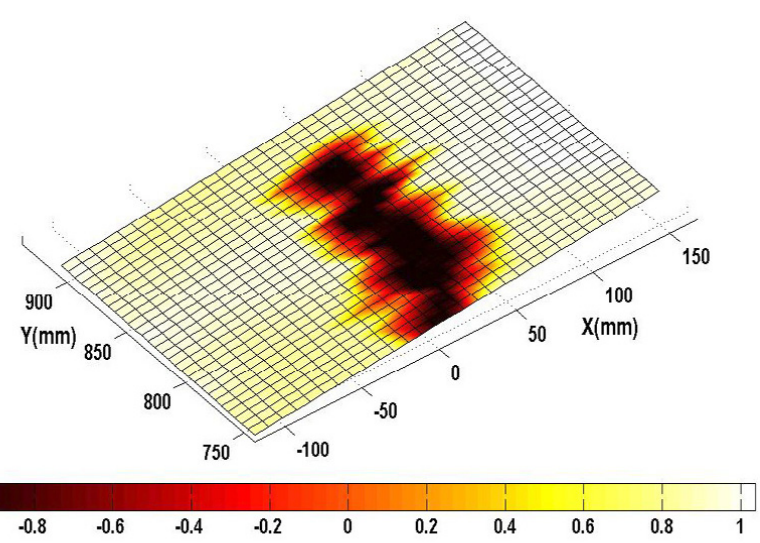

(a)

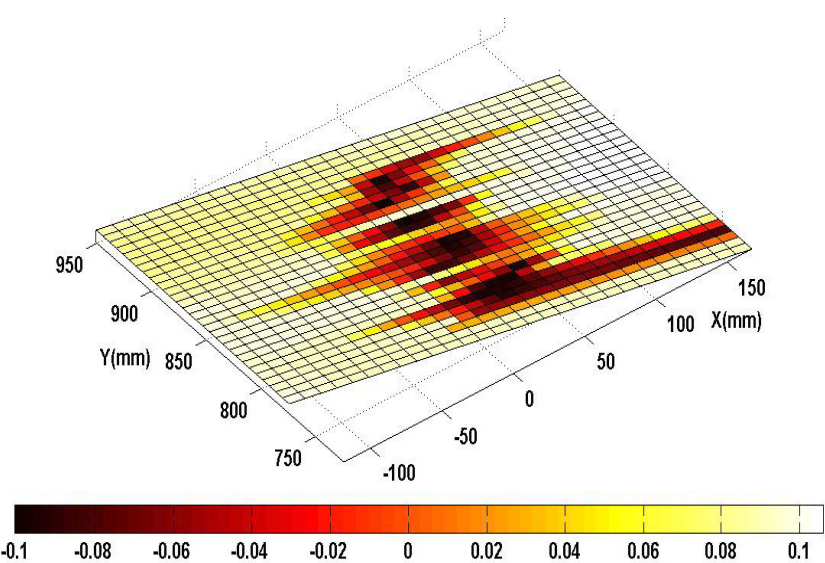

(b)

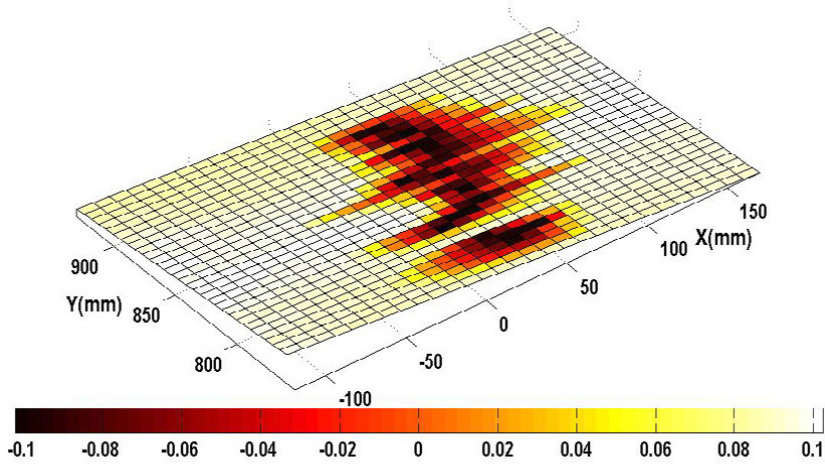

(c)

Fig. 4. Evaluated MDZs for randomly selected 200 measured points: (a) data set \#1, (b) data set \#7, (c) data set \#9.

seen in Table 3, the standard deviation of the evaluated MDZs using the convex hull approach for 9 cases is about $36.23 \%$ of the range of observations. This is a concerning observation since it demonstrates even if there is no computational uncertainty in the fitting process, the uncertainty due to sampling and plug-in nature of coordinate metrology can make the inspection process unreliable.

The error associated with using an optimization-based fitting algorithm is calculated by comparing its evaluations with the evaluations achieved using the convex hull approach. The goal here is not to study the efficiency of the 
Table 2. MDZs, normal vectors, fitting uncertainties, inspection errors due to sampling of 9 random sampling experiments with sample sizes of 200 data points-Units in columns $2,3,6$ and 7 are in millimetres.

\begin{tabular}{|c|c|c|c|c|c|c|}
\hline $\begin{array}{l}\text { Sampling } \\
\text { experiment }\end{array}$ & $\begin{array}{c}\text { MDZ: } \\
\text { convex hull }\end{array}$ & $\begin{array}{c}\text { MDZ: } \\
\text { optimization-based } \\
\text { fitting }\end{array}$ & $\begin{array}{l}\text { Normal vector: } \\
\text { convex hull }\end{array}$ & $\begin{array}{c}\text { Normal vector: } \\
\text { optimization-based } \\
\text { fitting }\end{array}$ & Fitting error & $\begin{array}{c}\text { Inspection error } \\
\text { due to sampling, } \%\end{array}$ \\
\hline 1 & \pm 0.1039 & \pm 0.1098 & $\begin{array}{r}0.0104 \\
-0.0056 \\
0.9999\end{array}$ & $\begin{array}{r}0.0091 \\
-0.0239 \\
0.9997\end{array}$ & 0.0060 & 10.76 \\
\hline 2 & \pm 0.1035 & \pm 0.1052 & $\begin{array}{r}0.0099 \\
-0.0050 \\
0.9999\end{array}$ & $\begin{array}{r}0.0113 \\
-0.0016 \\
0.9999\end{array}$ & 0.0017 & 11.11 \\
\hline 3 & \pm 0.1050 & \pm 0.1099 & $\begin{array}{r}0.0110 \\
-0.0007 \\
0.9999\end{array}$ & $\begin{array}{r}0.0157 \\
-0.0170 \\
0.9997\end{array}$ & 0.0048 & 9.78 \\
\hline 4 & \pm 0.1070 & \pm 0.1071 & $\begin{array}{r}0.0105 \\
-0.0040 \\
0.9999\end{array}$ & $\begin{array}{l}0.0122 \\
0.0001 \\
0.9999\end{array}$ & 0.00002 & 8.02 \\
\hline 5 & \pm 0.1098 & \pm 0.1063 & $\begin{array}{r}0.0101 \\
-0.0131 \\
0.9999\end{array}$ & $\begin{array}{r}0.0111 \\
-0.0205 \\
0.9997\end{array}$ & 0.0035 & 8.67 \\
\hline 6 & \pm 0.1055 & \pm 0.1045 & $\begin{array}{r}0.0094 \\
-0.0037 \\
1.0000\end{array}$ & $\begin{array}{r}0.0097 \\
-0.0003 \\
0.9999\end{array}$ & 0.0010 & $\% 10.2478$ \\
\hline 7 & \pm 0.1104 & \pm 0.1031 & $\begin{array}{r}0.0092 \\
-0.0024 \\
1.0000\end{array}$ & $\begin{array}{r}0.0091 \\
-0.0245 \\
0.9997\end{array}$ & 0.0073 & 11.42 \\
\hline 8 & \pm 0.1054 & \pm 0.1025 & $\begin{array}{r}0.0074 \\
-0.0085 \\
0.9999\end{array}$ & $\begin{array}{r}0.0109 \\
-0.0124 \\
0.9999\end{array}$ & 0.0028 & 11.92 \\
\hline 9 & \pm 0.1087 & \pm 0.1064 & $\begin{array}{r}0.0089 \\
-0.0194 \\
0.9998\end{array}$ & $\begin{array}{r}0.0126 \\
-0.0050 \\
0.9999\end{array}$ & 0.0023 & 8.56 \\
\hline
\end{tabular}

Table 3. Uncertainties in evaluation of MDZ of sample points.

\begin{tabular}{|c|c|c|c|c|}
\hline Observations & $\begin{array}{c}\text { Max.-Min. of } \\
\text { observations } \\
(\mathrm{mm})\end{array}$ & $\begin{array}{c}\text { Mean of } \\
\text { observations } \\
(\mathrm{mm})\end{array}$ & $\begin{array}{c}\text { Uncertainty in } \\
\text { observations } \\
(\mathrm{mm})\end{array}$ & $\begin{array}{c}\text { Uncertainty \% } \\
/ \\
\text { (Max.-Min.) }\end{array}$ \\
\hline MDZs by convex hull approach & 0.0069 & 0.1066 & 0.0025 & 36.23 \\
\hline $\begin{array}{c}\text { MDZs by } \\
\text { optimization-based fitting }\end{array}$ & 0.0074 & 0.1061 & 0.0026 & 35.30 \\
\hline $\begin{array}{c}\text { Error in } \\
\text { optimization-based fitting }\end{array}$ & 0.0073 & 0.0033 & 0.0024 & 32.73 \\
\hline $\begin{array}{c}\text { Error in MDZs of } \\
\text { sampling experiments } \\
\text { by convex hull } \\
\text { (comparing to the exact answer) }\end{array}$ & 0.0069 & 0.0098 & 0.0025 & 36.72 \\
\hline
\end{tabular}

utilized optimization-based fitting algorithm. The desire is to see the significance effect of sampling and it is seen the effect of sampling is as significant as the evaluation error in the utilized optimization-based fitting algorithm. The uncertainty of the fitting error over the range of error is about $32.73 \%$. Although this method has its own inherent source of error but changing the sample set of data points has almost the same significance of effect on the accuracy of the results that it had for the case of using convex hull approach (35.30\% versus $36.23 \%)$.

The last important observation in Tables 2 and 3 is about analysing the success level of the sampling attempts in evaluation of the real MDZ. It can be seen in Table 2 that in average all difference sampling produced $10.055 \%$ under-estimation of the MDZ. In addition, uncertainty of this error over the range of error is about $36.72 \%$. 


\section{Conclusion}

The sampling plan affects directly on both aspects of uncertainty and accuracy of coordinate metrology process. It is shown in this paper how changing the sample sets with the same density of data set changes the inspection results. As a main observation, uncertainty of inspection results due to change of locations of sample date points is observed to be as large as $36 \%$ of the range of deviations of the results. Examining the accuracy of the inspection results based on the sampling data shows that in all conducted experiments about $10 \%$ in average the MDZ is under-estimated. Understanding the risks and sources of uncertainties due to the density and type of sampling is crucial for industrial application. Ignoring these levels of uncertainties and the resulting lack of accuracy in the conducted inspection process may results in wrong acceptance of failing parts or false rejection of an acceptable product. Utilizing dynamic minimum deviation zone evaluation practiced in the paper can be an approach in developing closed-loops between the computational tasks required for fitting the optimum geometry to the data points and the adaptive sampling strategy, when the adaptive sampling process dynamically updates the data sets to enhance or examine the certainty of evaluated MDZ.

Acknowledgements. The research support provided by the Natural Science and Engineering Research Council of Canada (NSERC) is greatly appreciated.

\section{References}

1. T. Hopp, Computational metrology, Manuf. Rev. 6, 295304 (1993)

2. A. Barari, H.A. ElMaraghy, G.K. Knopf, Search-guided sampling to reduce uncertainty of minimum zone estimation, J. Comput. Inf. Sci. Eng. 7, 360-371 (2007)

3. W. Choi, T.R. Kurfess, J. Cagan, Sampling uncertainty in coordinate measurement data analysis, Precis. Eng. 22, 153-163 (1998)

4. H.A. ElMaraghy, A. Barari, G.K. Knopf, Integrated inspection and machining for maximum conformance to design tolerances, CIRP Ann. 53, 411-416 (2004)

5. R. Hocken, J. Raja, U. Babu, Sampling issue in coordinate metrology, Manuf. Rev. 6, 282-294 (1993)

6. G. Caskey, Y. Hari, R. Hocken, D. Palanvelu, J. Raja, R. Wilson, K. Chen, J. Yang, Sampling techniques for coordinate measuring machines, in Proc. of NSF Design ES Manufacturing Systems Conference, Austin, TX, USA, 1991, pp. 983-988

7. A. Weckenmann, H. Eitzert, M. Garmer, H. Webert, Functionality oriented evaluation and sampling strategy in coordinate metrology, Precis. Eng. 17, 244-251 (1995)

8. R. Edgeworth, R.G. Wilhelm, Adaptive sampling for coordinate metrology, Precis. Eng. 23, 144-154 (1999)

9. M.T. Traband, S. Joshi, R.A. Wysk, T.M. Carvalier, Evaluation of straightness and flatness tolerances using the minimum zone, Manuf. Rev. 2, 189-195 (1989)

10. J. O'Rourke, Computational Geometry in C, 2nd edn. (Cambridge University Press, New York, 1998), pp. $182-192$ 